\title{
New cosmological constraints with extended-Baryon Oscillation Spectroscopic Survey DR14 quasar sample
}

\author{
Lu Chen ${ }^{1,2, a}$, Qing-Guo Huang ${ }^{1,2,3, b}$, Ke Wang ${ }^{1,2, c}$ \\ ${ }^{1}$ CAS Key Laboratory of Theoretical Physics, Institute of Theoretical Physics, Chinese Academy of Sciences, Beijing 100190, China \\ ${ }^{2}$ School of Physical Sciences, University of Chinese Academy of Sciences, No. 19A Yuquan Road, Beijing 100049, China \\ ${ }^{3}$ Synergetic Innovation Center for Quantum Effects and Applications, Hunan Normal University, Changsha 410081, China
}

Received: 16 July 2017 / Accepted: 1 November 2017 / Published online: 13 November 2017

(C) The Author(s) 2017. This article is an open access publication

\begin{abstract}
We update the constraints on the cosmological parameters by adopting the Planck data released in 2015 and baryon acoustic oscillation (BAO) measurements including the new DR14 quasar sample measurement at redshift $z=1.52$, and we conclude that the six-parameter $\Lambda \mathrm{CDM}$ model is preferred. Exploring some extensions to the $\Lambda \mathrm{CDM}$ model, we find that the equation of state of dark energy reads $w=-1.036 \pm 0.056$ in the $w \mathrm{CDM}$ model, the effective number of relativistic degrees of freedom in the Universe is $N_{\text {eff }}=3.09_{-0.20}^{+0.18}$ in the $N_{\text {eff }}+\Lambda \mathrm{CDM}$ model and the spatial curvature parameter is $\Omega_{k}=(1.8 \pm 1.9) \times 10^{-3}$ in the $\Omega_{k}+\Lambda$ CDM model at $68 \%$ confidence level (C.L.), and the $95 \%$ C.L. upper bounds on the sum of three active neutrinos masses are $\sum m_{v}<0.16 \mathrm{eV}$ for the normal hierarchy $(\mathrm{NH})$ and $\sum m_{v}<0.19 \mathrm{eV}$ for the inverted hierarchy (IH) with $\Delta \chi^{2} \equiv \chi_{\mathrm{NH}}^{2}-\chi_{\mathrm{IH}}^{2}=-1.25$
\end{abstract}

\section{Introduction}

The accuracy of cosmological observations has been significantly improved in the past two decades. The six-parameter $\Lambda \mathrm{CDM}$ model is strongly supported by the precise measurements of anisotropies of the cosmic microwave background (CMB) [1,2]. The Type Ia supernova (SNe) $[3,4]$ and baryon acoustic oscillation (BAO) data $[5,6]$ as a geometric complement directly encode the information of the history of the expansion in the late-time Universe. As an important parameter characterizing today's expansion rate, the Hubble constant is directly measured by the Hubble space telescope (HST) [7].

\footnotetext{
a e-mail: chenlu@itp.ac.cn

b e-mail: huangqg@itp.ac.cn

c e-mail: wangke@itp.ac.cn
}

The BAO measurement is the periodic relic of fluctuations of baryonic matter density in the Universe. It is considered as a standard ruler of the Universe and can be used as an independent way to constrain models. In the previous observations, the BAO is traced directly by galaxies at low redshift and measured indirectly by analysis of the Lyman- $\alpha$ $(\operatorname{Ly} \alpha)$ forest in quasar spectra at high redshifts. Recently, the extended-Baryon Oscillation Spectroscopic Survey (eBOSS) [8] released their another percent level BAO measurement at $z=1.52$ using the auto-correlation of quasars directly, referred to as DR14 quasar sample [9]. It is a new method to achieve BAO features, which makes DR14 the first BAO distance observations in the range of $1<z<2$.

The higher redshift at which BAO is measured, the more sensitive to the Hubble parameter. Therefore, we can expect an improvement in constraints on the equation of state (EOS) of dark energy (DE) and a preciser description of the expansion history by including DR14. On the other hand, with increasing total active neutrino mass at fixed $\theta_{*}$, the spherically averaged BAO distance $D_{V}(z \lesssim 1)$ increases accordingly, but $D_{V}(z>1)$ falls [10]. It implies that DR14 may improve the constraint on the total active neutrino mass. On the contrary, with increasing the effective number of relativistic species $N_{\text {eff }}$ at a fixed $\theta_{*}$ and a fixed redshift of matterradiation equality $z_{\mathrm{eq}}, D_{V}(z)$ decreases for all BAO measurements [10]. Therefore, DR14 can improve the constraint on $N_{\text {eff }}$ as well. In addition, the spatial curvature of our Universe can also be constrained better because the geometry of space affects the detection of the BAO measurement directly and the new released BAO measurement DR 14 fills the gap between $1<z<2$

In this paper, we update the constraints on the EOS of DE, the active neutrino masses, the dark radiation and the spatial curvature with the Planck data and the BAO measurements including the DR14 quasar sample at $z=1.52$. The paper is arranged as follows. In Sect. 2, we explain our methodology 
and the data we used. In Sect. 3, the results for different models are presented. Finally, a brief summary and discussion are included in Sect. 4.

\section{Data and methodology}

We use the combined data of $\mathrm{CMB}$ and $\mathrm{BAO}$ measurements to constrain the parameters in the different models. Concretely, we use Planck TT,TE,EE+lowP released by the Planck Collaboration in 2015 [2], namely P15, as well as the BAO measurements at $z=0.106,0.15,0.32,0.57,1.52$, namely 6dFGS [11], MGS [12], DR12 BOSS LOWZ, DR12 BOSS CMASS [13,14], and DR14 eBOSS quasar sample [9] separately.

To show the BAO data we used, we should introduce the BAO model briefly, which is the basic model of the BAO signal. The volume-averaged values are measured, in [6], by

$D_{V}(z)=\left[(1+z)^{2} D_{A}^{2}(z) \frac{c z}{H(z)}\right]^{1 / 3}$

where $c$ is the light speed, $D_{A}(z)$ is the proper angular diameter distance [15], given by

$D_{A}(z)=\frac{c}{1+z} S_{k}\left(\int_{0}^{z} \frac{\mathrm{d} z^{\prime}}{H\left(z^{\prime}\right)}\right)$

where $S_{k}(x)$ is

$S_{k}(x)= \begin{cases}\sin \left(\sqrt{-\Omega_{k}} x\right) / \sqrt{-\Omega_{k}}, & \Omega_{k}<0, \\ \sinh \left(\sqrt{\Omega_{k}} x\right) / \sqrt{\Omega_{k}}, & \Omega_{k}>0, \\ x & \Omega_{k}=0,\end{cases}$

and $H(z)$ is

$$
\begin{aligned}
H(z)= & H_{0}\left[\Omega_{r}(1+z)^{4}+\Omega_{m}(1+z)^{3}+\Omega_{k}(1+z)^{2}\right. \\
& \left.+\left(1-\Omega_{r}-\Omega_{m}-\Omega_{k}\right) f(z)\right]^{1 / 2}
\end{aligned}
$$

where

$f(z) \equiv \frac{\rho_{\mathrm{DE}}(z)}{\rho_{\mathrm{DE}}(0)}=\exp \left[\int_{0}^{z} 3\left(1+w\left(z^{\prime}\right)\right) \frac{\mathrm{d} z^{\prime}}{1+z^{\prime}}\right]$,

and $w(z) \equiv p_{\mathrm{DE}} / \rho_{\mathrm{DE}}$ is the $\mathrm{EOS}$ of $\mathrm{DE}$.

\section{Results}

In this section, we will represent our new constraints on the dark energy, the neutrino masses, the dark radiation and the spatial curvature of the Universe separately.

\subsection{Constraints on dark energy}

In this subsection, we constrain the cosmological parameters in the $\Lambda \mathrm{CDM}$ model, the $w \mathrm{CDM}$ model and the $w_{0} w_{a} \mathrm{CDM}$ model $[16,17]$, respectively. Our results are summarized in Table 1. We run CosmoMC [18] in the $\Lambda$ CDM model as the basic model, where there are six free cosmological parameters $\left\{\Omega_{b} h^{2}, \Omega_{c} h^{2}, 100 \theta_{M C}, \tau, n_{s}, \ln \left(10^{10} A_{s}\right)\right\}$. Here $\Omega_{b} h^{2}$ is the density of the baryonic matter today, $\Omega_{c} h^{2}$ is the cold dark matter density today, $100 \theta_{M C}$ is 100 times the ratio of the angular diameter distance to the large scale structure sound horizon, $\tau$ is the optical depth, $n_{s}$ is the scalar spectrum index, and $A_{s}$ is the amplitude of the power spectrum of primordial curvature perturbations.

The EOS of DE is $w=-1.036 \pm 0.056$ in the $w \mathrm{CDM}$ model at $68 \%$ confidence level (C.L.). The triangular plot of $H_{0}, w_{0}$ and $w_{a}$ in the $w_{0} w_{a}$ CDM model is shown in Fig. 1 and it indicates that the prediction of $\Lambda \mathrm{CDM}$ is within the $68 \%$ confidence region in this figure, which seems to be in conflict with the $w_{0}, w_{a}$ values in Table 1. Actually, the probabilities are the integrated probabilities, which means the values in Table 1 have been marginalized over all the other parameters except the aimed parameter. Due to the strong correlation between $w_{0}$ and $w_{a}$, we should check if the prediction of $\Lambda \mathrm{CDM}$ is consistent with datasets in the $w_{0}-w_{a} 2 \mathrm{D}$ contour plot.

Marginalizing over the other cosmological parameters, we also plot the evolution of the normalized Hubble parameter $H(z)$ in Fig. 2 where the Hubble parameter is normalized by comparing with those in the best-fit $\Lambda \mathrm{CDM}$ model.

The Hubble constant is $H_{0}=67.81_{-0.46}^{+0.47} \mathrm{~km} \mathrm{~s}^{-1} \mathrm{Mpc}^{-1}$ in the $\Lambda \mathrm{CDM}$ model, $H_{0}=68.66_{-1.55}^{+1.41} \mathrm{~km} \mathrm{~s}^{-1} \mathrm{Mpc}^{-1}$ in the $w \mathrm{CDM}$ model, and $H_{0}=62.56_{-2.74}^{+2.42} \mathrm{~km} \mathrm{~s}^{-1} \mathrm{Mpc}^{-1}$ in the $w_{0} w_{a} \mathrm{CDM}$ model, at $68 \%$ C.L. In all, there is a significant tension on the measurement of Hubble constant between global fitting P15+BAO and the direct measurement by HST in [7] (named R16) which gives $H_{0}=73.24 \pm 1.74 \mathrm{~km} \mathrm{~s}^{-1}$ $\mathrm{Mpc}^{-1}$. Even though such a tension is slightly relaxed in the $w \mathrm{CDM}$ model, it is aggravated in the $w_{0} w_{a} \mathrm{CDM}$ model. In order to significantly relax such a tension, a more dramatic design of the EOS of DE is needed [19]. In addition, a tension still exists around $2 \sigma$ on the Hubble parameter at $z=2.34$ between the predictions of these three DE models constrained by P15+BAO and the measurement by Ly $\alpha$ forest of BOSS DR11 quasars [20], which gives $H(z=2.34)=222 \pm 7 \mathrm{~km}$ $\mathrm{s}^{-1} \mathrm{Mpc}^{-1}$.

\subsection{Constraints on the total mass of active neutrinos}

The neutrino oscillation implies that the active neutrinos have mass splittings

$$
\begin{gathered}
\Delta m_{21}^{2}=m_{2}^{2}-m_{1}^{2}, \\
\left|\Delta m_{31}^{2}\right|=\left|m_{3}^{2}-m_{1}^{2}\right| .
\end{gathered}
$$


Table 1 The $68 \%$ limits for the cosmological parameters in different DE models from $\mathrm{P} 15+\mathrm{BAO}$

\begin{tabular}{llll}
\hline & $\Lambda \mathrm{CDM}$ & $w \mathrm{CDM}$ & $w_{0} w_{a} \mathrm{CDM}$ \\
\hline$\Omega_{b} h^{2}$ & $0.02233 \pm 0.00014$ & $0.02229 \pm 0.00015$ & $0.02224 \pm 0.00015$ \\
$\Omega_{c} h^{2}$ & $0.1186 \pm 0.0010$ & $0.1191 \pm 0.0013$ & $0.1200 \pm 0.0014$ \\
$100 \theta_{M C}$ & $1.04091 \pm 0.00030$ & $1.04086 \pm 0.00031$ & $1.04075 \pm 0.00031$ \\
$\tau$ & $0.085 \pm 0.016$ & $0.082 \pm 0.017$ & $0.075 \pm 0.017$ \\
$\ln \left(10^{10} A_{s}\right)$ & $3.102 \pm 0.032$ & $3.098_{-0.032}^{+0.035}$ & $3.086 \pm 0.033$ \\
$n_{s}$ & $0.9677 \pm 0.0040$ & $0.9664 \pm 0.0046$ & $0.9640 \pm 0.0046$ \\
$w$ & - & $-1.036 \pm 0.056$ & - \\
$w_{0}$ & - & - & $-0.25 \pm 0.32$ \\
$w_{a}$ & - & - & $-2.29_{-0.91}^{+1.10}$ \\
$H_{0}\left(\mathrm{~km} \mathrm{~s}^{-1} \mathrm{Mpc}^{-1}\right)$ & $67.81_{-0.46}^{+0.47}$ & $68.66_{-1.55}^{+1.41}$ & $62.56_{-2.74}^{+2.42}$
\end{tabular}

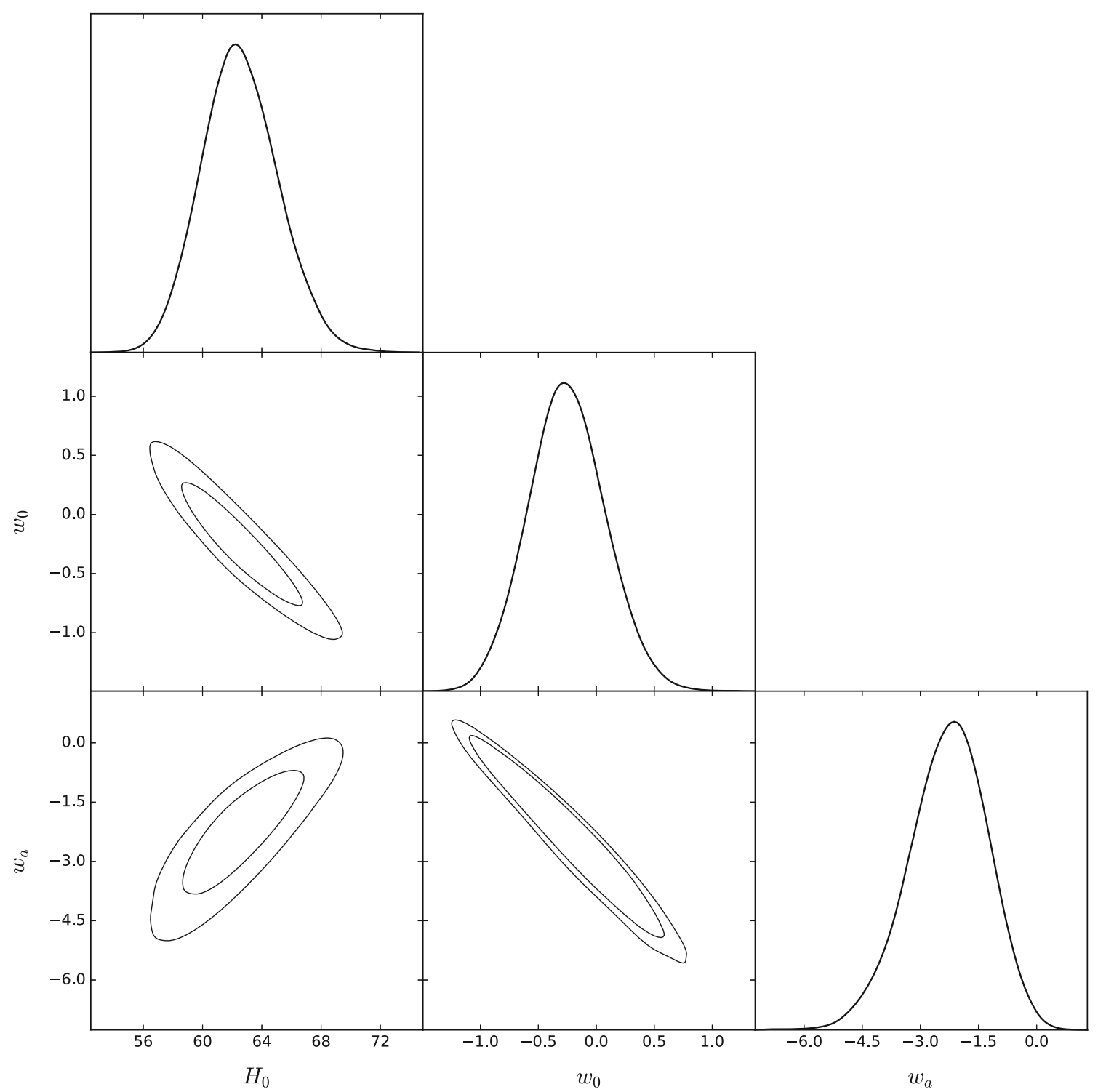

Fig. 1 The triangular plot of $H_{0}, w_{0}$ and $w_{a}$ in the $w_{0} w_{a}$ CDM model. The point $\left(w_{0}=-1, w_{a}=0\right)$ locates in the $w_{0} w_{a}$ CDM $68 \%$ C.L. region 


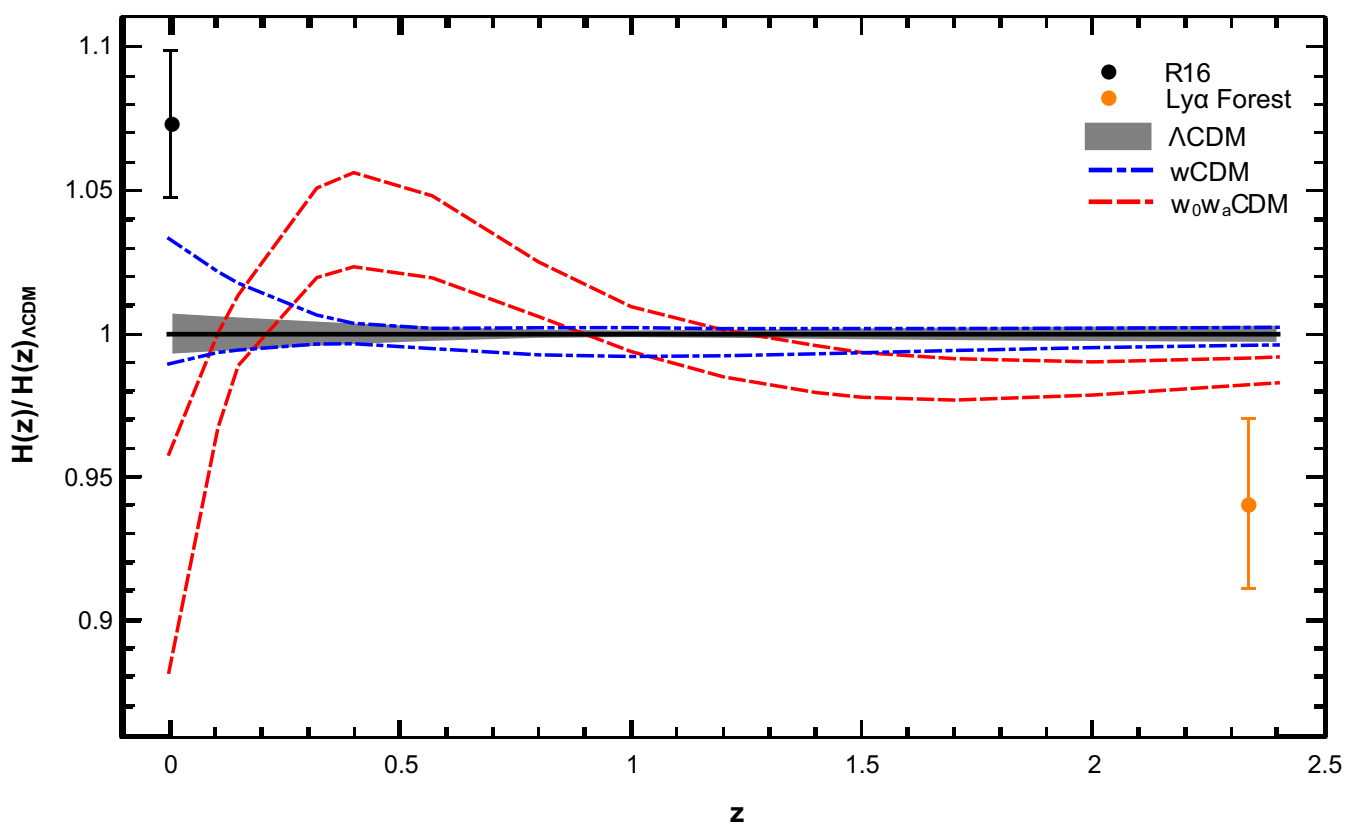

Fig. 2 The normalized $H(z)$ plot in the $\Lambda$ CDM model, $w$ CDM model and $w_{0} w_{a} \mathrm{CDM}$ model. The gray band represents the $68 \%$ confidence range in the $\Lambda \mathrm{CDM}$ model allowed by $\mathrm{P} 15+\mathrm{BAO}$. The ranges between the two blue dash-dotted lines and the two red dashed lines represent

Table 2 The $68 \%$ limits for the cosmological parameters in the $v_{\mathrm{NH}} \Lambda \mathrm{CDM}$ model and the $v_{\mathrm{IH}} \Lambda \mathrm{CDM}$ model from P15+BAO

\begin{tabular}{lll}
\hline & $\nu_{\mathrm{NH}} \Lambda \mathrm{CDM}$ & $\nu_{\mathrm{IH}} \Lambda \mathrm{CDM}$ \\
\hline$\Omega_{b} h^{2}$ & $0.02234 \pm 0.00014$ & $0.02235 \pm 0.00014$ \\
$\Omega_{c} h^{2}$ & $0.1184 \pm 0.0011$ & $0.1181 \pm 0.0010$ \\
$100 \theta_{M C}$ & $1.04093 \pm 0.00029$ & $1.04093 \pm 0.00029$ \\
$\tau$ & $0.087 \pm 0.0165$ & $0.090_{-0.016}^{+0.018}$ \\
$\ln \left(10^{10} A_{s}\right)$ & $3.106 \pm 0.033$ & $3.111_{-0.032}^{+0.035}$ \\
$n_{s}$ & $0.9682_{-0.0040}^{+0.0041}$ & $0.9689 \pm 0.0041$ \\
$m_{v, \text { min }}(95 \%$ C.L. $)$ & $<0.047 \mathrm{eV}$ & $<0.049 \mathrm{eV}$ \\
$\Sigma m_{v}(95 \%$ C.L. $)$ & $<0.16 \mathrm{eV}$ & $<0.19 \mathrm{eV}$ \\
$H_{0}\left(\mathrm{~km} \mathrm{~s}^{-1} \mathrm{Mpc}^{-1}\right)$ & $67.65 \pm 0.50$ & $67.43 \pm 0.48$ \\
\hline
\end{tabular}

where $\Delta m_{21}^{2} \simeq 7.54 \times 10^{-5} \mathrm{eV}^{2}$ and $\left|\Delta m_{31}^{2}\right| \simeq 2.46 \times$ $10^{-3} \mathrm{eV}^{2}$ [21]. That is to say, there are two possible mass hierarchies: if $m_{1}<m_{2}<m_{3}$, it is a normal hierarchy (NH); if $m_{3}<m_{1}<m_{2}$, it is an inverted hierarchy (IH).

The neutrino mass spectrum is expressed as the $68 \%$ confidence ranges of the $w \mathrm{CDM}$ model and $w_{0} w_{a} \mathrm{CDM}$ model allowed by $\mathrm{P} 15+\mathrm{BAO}$, respectively. The black and orange error bars denote the Hubble parameters measured by HST (named R16) in [7] and the Ly $\alpha$ forest of BOSS DR11 quasars (named Ly $\alpha$ Forest) in [20]

and the total mass satisfies

$\sum m_{v}=m_{1}+m_{2}+m_{3} \gtrsim \begin{cases}0.059, & \text { for } \mathrm{NH}, \\ 0.101, & \text { for } \mathrm{IH} .\end{cases}$

Here we set the minimum of the three neutrino masses as a free parameter and the sum of the neutrino masses as a derived parameter. Our results are summarized in Table 2.

The likelihood distribution of $\sum m_{v}$ for the $\mathrm{NH}$ and $\mathrm{IH}$ are illustrated in Fig. 3.

In summary, the masses of the lightest neutrinos in $\mathrm{NH}$ and $\mathrm{IH}$ are $m_{v, \text { min }}<0.047 \mathrm{eV}$ and $m_{v, \text { min }}<0.049 \mathrm{eV}$ at 95\% C.L. respectively. The total active neutrino masses are given by $\sum m_{v}<0.16 \mathrm{eV}$ and $\sum m_{v}<0.19 \mathrm{eV}$ for the $\mathrm{NH}$ and $\mathrm{IH}$, and the $\mathrm{NH}$ is slightly preferred with $\Delta \chi^{2} \equiv$ $\chi_{\mathrm{NH}}^{2}-\chi_{\mathrm{IH}}^{2}=-1.25$. Our new results are slightly tighter than those without the DR14 quasar sample [22]. For other related investigations we refer to [23-31].

\subsection{Constraints on the dark radiation}

The total energy density of radiation in the Universe is given by

$\left(m_{1}, m_{2}, m_{3}\right)= \begin{cases}\left(m_{1}, \sqrt{m_{1}^{2}+\Delta m_{21}^{2}}, \sqrt{m_{1}^{2}+\left|\Delta m_{31}^{2}\right|}\right), & \text { for NH, where } m_{1} \text { is the minimum, } \\ \left(\sqrt{m_{3}^{2}+\left|\Delta m_{31}^{2}\right|}, \sqrt{m_{3}^{2}+\Delta m_{21}^{2}+\left|\Delta m_{31}^{2}\right|}, m_{3}\right), & \text { for IH, where } m_{3} \quad \text { is the minimum, }\end{cases}$ 


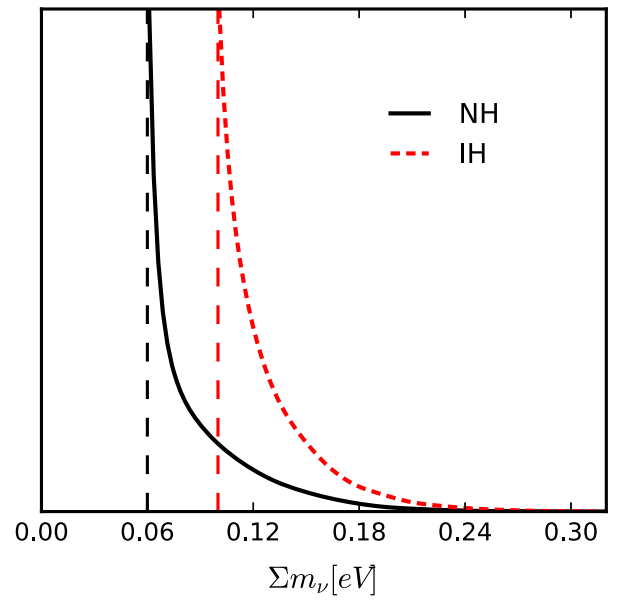

Fig. 3 The likelihood distributions of $\sum m_{v}$ for the NH and IH neutrinos in the $v \Lambda \mathrm{CDM}$ model. The dashed lines donate the allowed minimums of $\sum m_{v}$, namely $0.059 \mathrm{eV}$ for $\mathrm{NH}$ and $0.101 \mathrm{eV}$ for $\mathrm{IH}$

$\rho_{r}=\left[1+\frac{7}{8}\left(\frac{4}{11}\right)^{4 / 3} N_{\text {eff }}\right] \rho_{\gamma}$

where $\rho_{\gamma}$ is the CMB photon energy density, $N_{\text {eff }}$ denotes the effective number of relativistic degrees of freedom in the Universe. For the three standard model neutrinos, their contribution to $N_{\text {eff }}$ is 3.046 due to non-instantaneous decoupling corrections. Then the additional relativistic degree of freedom $\Delta N_{\text {eff }} \equiv N_{\text {eff }}-3.046$ implies the existence of some other unknown sources of relativistic degree of freedom. $\Delta N_{\text {eff }}<0$ is considered to result from incompletely thermalized neutrinos or the existence of photons produced after neutrino decoupling, which is less motivated. But there exist many cases with $\Delta N_{\text {eff }}>0$. If a kind of additional massless particles don't interact with others since the epoch of recombination, their energy density evolves exactly like radiation and thus contributes $\Delta N_{\text {eff }}=1$. There are more explanation for $0<\Delta N_{\text {eff }}<1$ considering the non-thermal case and the bosonic particles. The thermalized massless boson decoupled during $0.5 \mathrm{MeV}<T<100 \mathrm{MeV}$ contributes $\Delta N_{\text {eff }} \simeq 0.57$ and $\Delta N_{\text {eff }} \simeq 0.39$ if they decoupled before $T=100 \mathrm{MeV}$ [32].

In the $N_{\text {eff }}+\Lambda \mathrm{CDM}$ model, $N_{\text {eff }}$ is taken as a free parameter. The results are summarized in Table 3 . Our results give $N_{\text {eff }}=3.09_{-0.20}^{+0.18}$ at $68 \%$ C.L., which is consistent with the fact that there are only three active neutrinos in the Universe. On the other hand, for example in [7], the dark radiation is proposed to relax the tension on the Hubble constant between the global fitting P15+BAO and the direct measurement by HST. Here we illustrate the constraints on $H_{0}$ and $N_{\text {eff }}$ in Fig. 4. From Fig. 4, we find that the dark radiation cannot really solve this tension.
Table 3 The $68 \%$ limits for the cosmological parameters in the $N_{\text {eff }}+$ $\Lambda$ CDM model from P15+BAO

\begin{tabular}{ll}
\hline & $N_{\text {eff }}+\Lambda \mathrm{CDM}$ \\
\hline$\Omega_{b} h^{2}$ & $0.02236 \pm 0.00019$ \\
$\Omega_{c} h^{2}$ & $0.1193 \pm 0.0031$ \\
$100 \theta_{M C}$ & $1.04085 \pm 0.00044$ \\
$\tau$ & $0.086 \pm 0.017$ \\
$\ln \left(10^{10} A_{s}\right)$ & $3.105 \pm 0.035$ \\
$n_{s}$ & $0.9691_{-0.0075}^{+0.0076}$ \\
$N_{\text {eff }}$ & $3.09_{-0.20}^{+0.18}$ \\
$H_{0}\left(\mathrm{~km} \mathrm{~s}^{-1} \mathrm{Mpc}^{-1}\right)$ & $68.07_{-1.20}^{+1.21}$ \\
\hline
\end{tabular}

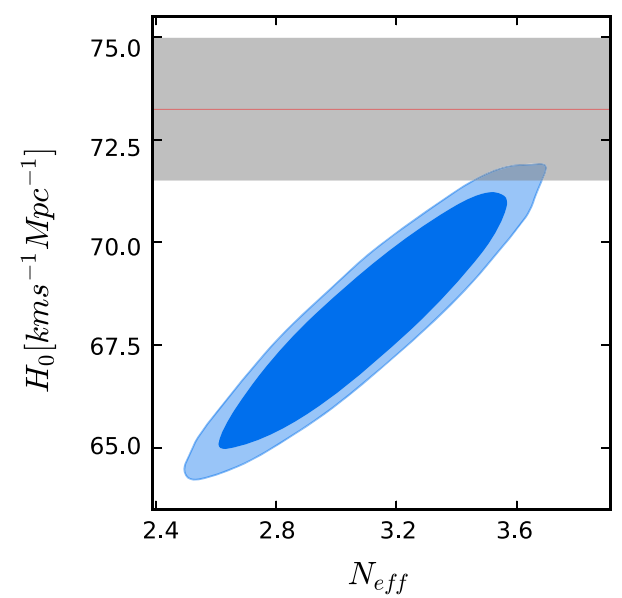

Fig. 4 The 2D contour plot of $H_{0}$ and $N_{\text {eff }}$ in the $N_{\text {eff }}+\Lambda C D M$ model. The rectangle shaded region represents the observational value $H_{0}=$ $73.24 \pm 1.74 \mathrm{~km} \mathrm{~s}^{-1} \mathrm{Mpc}^{-1}$. They are overlapped at the 95\% C.L. region

\subsection{Constraints on the curvature}

According to Eq. (2), the spatial geometry affects the distance measurements, and hence the spatial curvature parameter $\Omega_{k}$ can be constrained by using BAO data. In the $\Omega_{k}+\Lambda \mathrm{CDM}$ model, $\Omega_{k}$ is taken as a free parameter. The constraints on the cosmological parameters in the $\Omega_{k}+\Lambda \mathrm{CDM}$ model are given in Table 4.

We find that the spatial curvature has been tightly constrained, namely $\Omega_{k}=(1.8 \pm 1.9) \times 10^{-3}$ at $68 \%$ C.L. and $\Omega_{k}=\left(1.8_{-3.8}^{+3.9}\right) \times 10^{-3}$ at $95 \%$ C.L. which is nicely consistent with a spatially flat Universe. Adopting P15 only, the constraint on the spatial curvature is $\Omega_{k}=\left(-40_{-41}^{+38}\right) \times 10^{-3}$ at $95 \%$ C.L. which is around one oder of magnitude looser compared with our new result. However, our results improves little compared with the Planck $+\mathrm{BAO}$ result in the Planck table, $\Omega_{k}=(0.2 \pm 2.1) \times 10^{-3}$ at $68 \%$ C.L., which implies that the DR14 sample helps little to constrain the curvature. The constraints on $\Omega_{\Lambda}$ and $\Omega_{m}$ are illustrated in Fig. 5 . 
Table 4 The $68 \%$ limits for the cosmological parameters in the $\Omega_{k}+$ $\Lambda \mathrm{CDM}$ model from $\mathrm{P} 15+\mathrm{BAO}$

\begin{tabular}{ll}
\hline & $\Omega_{k}+\Lambda \mathrm{CDM}$ \\
\hline$\Omega_{b} h^{2}$ & $0.02226 \pm 0.00016$ \\
$\Omega_{c} h^{2}$ & $0.1196 \pm 0.0015$ \\
$100 \theta_{M C}$ & $1.04078 \pm 0.00030$ \\
$\tau$ & $0.081 \pm 0.017$ \\
$\ln \left(10^{10} A_{s}\right)$ & $3.097 \pm 0.033$ \\
$n_{s}$ & $0.9652 \pm 0.0048$ \\
$\Omega_{k}$ & $(1.8 \pm 1.9) \times 10^{-3}$ \\
$H_{0}\left(\mathrm{~km} \mathrm{~s}^{-1} \mathrm{Mpc}^{-1}\right)$ & $68.27_{-0.95}^{+0.68}$ \\
\hline
\end{tabular}

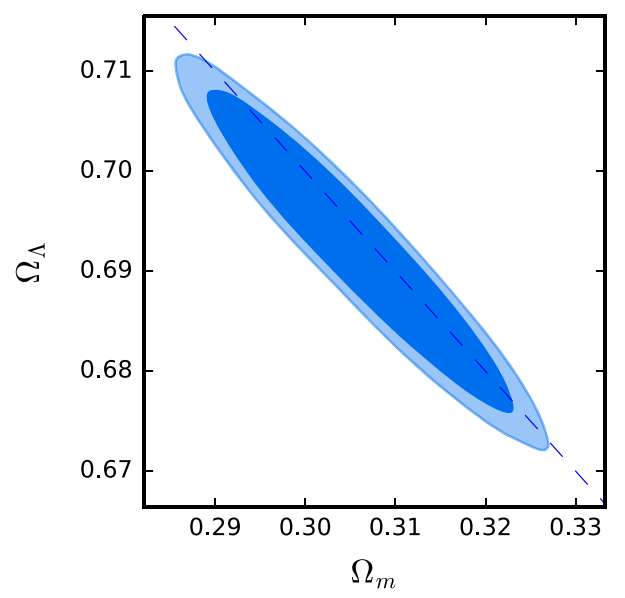

Fig. 5 The contour plot of $\Omega_{m}$ and $\Omega_{\Lambda}$ in the $\Omega_{k}+\Lambda$ CDM model. The dashed line indicates $\Omega_{m}+\Omega_{\Lambda}=1$

\section{Summary and discussion}

In this paper we provide the new constraints on the cosmological parameters in some extensions to the six-parameter $\Lambda \mathrm{CDM}$ model by combining P15 and BAO data including the DR14 quasar sample measurement released recently by eBOSS. We do not find any signals beyond this cosmological model.

We explore the EOS of DE in two extended models, namely $w \mathrm{CDM}$ and $w_{0} w_{a} \mathrm{CDM}$ model, and find $w=$ $-1.036 \pm 0.056$ at $68 \%$ C.L. in the $w \mathrm{CDM}$ model, $w_{0}=$ $-0.25 \pm 0.32, w_{a}=-2.29_{-0.91}^{+1.10}$ at $68 \%$ C.L. in the $w_{0} w_{a} \mathrm{CDM}$ model and $w=-1$ is located within the $68 \%$ C.L. region. But the tension on the Hubble constant with the direct measurement by HST and the global fitting P15+BAO in $w \mathrm{CDM}$ model cannot be significantly relaxed and the $w_{0} w_{a} \mathrm{CDM}$ model makes even worse. The neutrino mass normal hierarchy is slightly preferred by $\Delta \chi^{2} \equiv \chi_{\mathrm{NH}}^{2}-\chi_{\mathrm{IH}}^{2}=$ -1.25 compared to the inverted hierarchy, and the $95 \%$ C.L. upper bounds on the sum of three active neutrinos masses are $\sum m_{v}<0.16 \mathrm{eV}$ for the normal hierarchy and $\sum m_{v}<$ $0.19 \mathrm{eV}$ for the inverted hierarchy. The three active neutrinos are nicely consistent with the constraint on the effective relativistic degrees of freedom with $N_{\text {eff }}=3.09_{-0.20}^{+0.18}$ at $68 \%$ C.L., and a spatially flat Universe is preferred.

Acknowledgements We acknowledge the use of HPC Cluster of ITPCAS. This work is supported by grants from NSFC (Grant Nos. 11335012, 11575271, 11690021), Top-Notch Young Talents Program of China, and partly supported by Key Research Program of Frontier Sciences, CAS.

Open Access This article is distributed under the terms of the Creative Commons Attribution 4.0 International License (http://creativecomm ons.org/licenses/by/4.0/), which permits unrestricted use, distribution, and reproduction in any medium, provided you give appropriate credit to the original author(s) and the source, provide a link to the Creative Commons license, and indicate if changes were made. Funded by SCOAP . $^{3}$

\section{References}

1. E. Komatsu et al. [WMAP Collaboration], Astrophys. J. Suppl. 180, 330 (2009). https://doi.org/10.1088/0067-0049/180/2/330. arXiv:0803.0547 [astro-ph]

2. P.A.R. Ade et al. [Planck Collaboration], Astron. Astrophys. 594, A13 (2016). https://doi.org/10.1051/0004-6361/201525830. arXiv:1502.01589 [astro-ph.CO]

3. A. Conley et al. [SNLS Collaboration], Astrophys. J. Suppl. 192, 1 (2011). https://doi.org/10.1088/0067-0049/192/1/1. arXiv:1104.1443 [astro-ph.CO]

4. N. Suzuki et al., Astrophys. J. 746, 85 (2012). https://doi.org/10. 1088/0004-637X/746/1/85. arXiv:1105.3470 [astro-ph.CO]

5. S. Cole et al. [2dFGRS Collaboration], Mon. Not. R. Astron. Soc. 362, 505 (2005). https://doi.org/10.1111/j.1365-2966.2005. 09318.x. arXiv:astro-ph/0501174

6. D.J. Eisenstein et al. [SDSS Collaboration], Astrophys. J. 633, 560 (2005). https://doi.org/10.1086/466512. arXiv:astro-ph/0501171

7. A.G. Riess et al., Astrophys. J. 826(1), 56 (2016). https://doi.org/ 10.3847/0004-637X/826/1/56. arXiv:1604.01424 [astro-ph.CO]

8. K.S. Dawson et al., Astron. J. 151, 44 (2016). https://doi.org/10. 3847/0004-6256/151/2/44. arXiv:1508.04473 [astro-ph.CO]

9. M. Ata et al., arXiv:1705.06373 [astro-ph.CO]

10. P.A.R. Ade et al., [Planck Collaboration], Astron. Astrophys. 571, A16 (2014). https://doi.org/10.1051/0004-6361/201321591. arXiv:1303.5076 [astro-ph.CO]

11. F. Beutler et al., Mon. Not. R. Astron. Soc. 416, 3017 (2011). https://doi.org/10.1111/j.1365-2966.2011.19250.x. arXiv:1106.3366 [astro-ph.CO]

12. A.J. Ross, L. Samushia, C. Howlett, W.J. Percival, A. Burden, M. Manera, Mon. Not. R. Astron. Soc. 449(1), 835 (2015). https://doi. org/10.1093/mnras/stv154. arXiv:1409.3242 [astro-ph.CO]

13. H. Gil-Marin et al., Mon. Not. R. Astron. Soc. 460(4), 4210 (2016). https://doi.org/10.1093/mnras/stw1264. arXiv:1509.06373 [astroph.CO]

14. A.J. Cuesta et al., Mon. Not. R. Astron. Soc. 457(2), 1770 (2016). https://doi.org/10.1093/mnras/stw066. arXiv:1509.06371 [astroph.CO]

15. Aubourg, Phys. Rev. D 92(12), 123516 (2015). https://doi.org/10. 1103/PhysRevD.92.123516. arXiv:1411.1074 [astro-ph.CO]

16. E.V. Linder, Phys. Rev. Lett. 90, 091301 (2003). https://doi.org/10. 1103/PhysRevLett.90.091301. arXiv:astro-ph/0208512

17. M. Chevallier, D. Polarski, Int. J. Mod. Phys. D 10, 213 (2001). https://doi.org/10.1142/S0218271801000822. arXiv:gr-qc/0009008 
18. A. Lewis, S. Bridle, Phys. Rev. D 66, 103511 (2002). https://doi. org/10.1103/PhysRevD.66.103511. arXiv:astro-ph/0205436

19. Q.G. Huang, K. Wang, Eur. Phys. J. C 76(9), 506 (2016). https://doi. org/10.1140/epjc/s10052-016-4352-x. arXiv:1606.05965 [astroph.CO]

20. T. Delubac et al. [BOSS Collaboration], Astron. Astrophys. 574, A59 (2015). https://doi.org/10.1051/0004-6361/201423969. arXiv:1404.1801 [astro-ph.CO]

21. C. Patrignani et al. [Particle Data Group], Chin. Phys. C 40(10), 100001 (2016). https://doi.org/10.1088/1674-1137/40/10/100001

22. Q.G. Huang, K. Wang, S. Wang, Eur. Phys. J. C 76(9), 489 (2016). https://doi.org/10.1140/epjc/s10052-016-4334-z. arXiv:1512.05899 [astro-ph.CO]

23. L. Xu, Q.G. Huang, arXiv:1611.05178 [astro-ph.CO]

24. R.Y. Guo, Y.H. Li, J.F. Zhang, X. Zhang, JCAP 1705(05), 040 (2017). https://doi.org/10.1088/1475-7516/2017/05/040. arXiv:1702.04189 [astro-ph.CO]

25. E. K. Li, H. Zhang, M. Du, Z. H. Zhou, L. Xu, arXiv:1703.01554 [astro-ph.CO]
26. F. Capozzi, E. Di Valentino, E. Lisi, A. Marrone, A. Melchiorri, A. Palazzo, Phys. Rev. D 95(9), 096014 (2017). https://doi.org/10. 1103/PhysRevD.95.096014. arXiv:1703.04471 [hep-ph]

27. L. Feng, J.F. Zhang, X. Zhang, Eur. Phys. J. C 77(6), 418 (2017). https://doi.org/10.1140/epjc/s10052-017-4986-3. arXiv:1703.04884 [astro-ph.CO]

28. L. Feng, J.F. Zhang, X. Zhang arXiv:1706.06913 [astro-ph.CO]

29. S. Wang, D.M. Xia, arXiv:1707.00588 [astro-ph.CO]

30. S. Vagnozzi, E. Giusarma, O. Mena, K. Freese, M. Gerbino, S. Ho, M. Lattanzi, arXiv:1701.08172 [astro-ph.CO]

31. E. Giusarma, M. Gerbino, O. Mena, S. Vagnozzi, S. Ho, K. Freese, Phys. Rev. D 94(8), 083522 (2016). https://doi.org/10. 1103/PhysRevD.94.083522. arXiv:1605.04320 [astro-ph.CO]

32. S. Weinberg, Phys. Rev. Lett. 110(24), 241301 (2013). https://doi. org/10.1103/PhysRevLett.110.241301. arXiv:1305.1971 [astroph.CO] 